\section{Cureus}

\title{
Diagnosing Allergic Bronchopulmonary Aspergillosis: A Review
}

\author{
Avani R. Patel ${ }^{1}$, Amar R. Patel ${ }^{1}$, Shivank Singh ${ }^{2,} 3$, Shantanu Singh ${ }^{4}$, Imran Khawaja ${ }^{4}$ \\ 1. Internal Medicine, Northern California Kaiser Permanente, Fremont, USA 2. Internal Medicine, \\ Southern Medical University, Guangzhou, CHN 3. Internal Medicine, Maoming People's Hospital, \\ Maoming, CHN 4. Pulmonary Medicine, Marshall University School of Medicine, Huntington, USA
}

Corresponding author: Avani R. Patel, avani.94539@gmail.com

\section{Abstract}

Dr. Hinson and his colleagues first described allergic bronchopulmonary aspergillosis (ABPA) in 1952. Later in 1977, Rosenberg proposed a diagnostic criteria for ABPA that even today remains widely acknowledged. Despite these steps taken, there still isn't a standardized diagnostic criteria set for ABPA although many have been proposed by various physicians over the years. ABPA is a condition caused by hypersensitivity to Aspergillus fumigatus antigens. It is seen most commonly in patients with either asthma or cystic fibrosis. In susceptible hosts, repeated inhalation of Aspergillus spores can cause an allergic response. Although a standardized diagnostic criteria is required, there is no single test that establishes the diagnosis other than a demonstration of central bronchiectasis (CB) with normal tapering bronchi, a feature that is still considered pathognomonic of ABPA. Because of lack of standardized diagnostic criteria and screening, even today ABPA is under diagnosed and often times treatment for it is delayed. This can lead to complications in patients like pulmonary fibrosis, bronchiectasis with chronic sputum production, and increasingly severe persistent asthma with loss of lung function. For this alone, it becomes imperative that the diagnostic criteria guidelines need to be reviewed and standardized preferably with the help of larger research studies. In the following review article, we address the epidemiology, pathophysiology, and the current cumulative view regarding the diagnosis of ABPA.

Categories: Allergy/Immunology, Infectious Disease, Pulmonology

Keywords: allergic bronchopulmonary aspergillosis, aspergillus fumigatus, central bronchiectasis, asthma, cystic fibrosis, eosinophilia, aspergillosis

\section{Introduction And Background}

Received 04/05/2019

Review began 04/12/2019

Review ended 04/16/2019

Published 04/27/2019

\section{(c) Copyright 2019}

Patel et al. This is an open access article distributed under the terms of the Creative Commons Attribution License CC-BY 3.0., which permits unrestricted use, distribution, and reproduction in any medium, provided the original author and source are credited.
Aspergillosis is a condition in which Aspergillus fungi infect tissues. Aspergillosis affecting the respiratory tract can have several manifestations that range from hypersensitivity disorders to rapidly invasive disseminated disease, all of which can be classified within three distinct categories [1-2]. They are allergic aspergillosis, saprophytic colonization, and invasive aspergillosis [3]. Allergic aspergillosis can be further subdivided into three different forms. They include allergic bronchopulmonary aspergillosis (ABPA), Aspergillus-induced asthma (AIA), and allergic Aspergillus sinusitis (AAS) [3]. ABPA is a condition caused by a hypersensitivity reaction to Aspergillus antigens [3]. ABPA is classified differently depending on the criteria that the patient meets [4]. Asthma patients who meet the minimum criteria but do not have central or peripheral bronchiectasis are classified as serologic ABPA or ABPA-seropositive (ABPA-S) [57]. Patients who meet the minimum criteria for ABPA and also have central bronchiectasis are classified as ABPA-central bronchiectasis (ABPA-CB) [5-7]. Lastly, patients who have severe asthma and sensitivity to fungi but do not meet the criteria for ABPA are classified as severe asthma associated with fungal sensitivity (SAFS) [5-7]. In susceptible hosts, an allergic response 


\section{Cureus}

is evoked by repeated inhalation of Aspergillus spores [3]. These spores get trapped in the thick sputum of asthmatic subjects causing a cascade of inflammatory reactions that can result in ABPA [3]. ABPA is found most commonly in atopic patients, or in patients with cystic fibrosis or asthma. The following review article addresses ABPA and its epidemiology, pathophysiology, and diagnostic criteria.

\section{Review}

\section{Epidemiology}

ABPA affects approximately $1 \%$ through $15 \%$ of cystic fibrosis patients [8]. One study calculated that $2.5 \%$ of adults who have asthma also have ABPA, which is approximately 4.8 million people worldwide [9]. From 1983 through 1986, Greenberger and Patterson found an ABPA prevalence of $6 \%$ from their 531 asthmatic patients [10]. In other studies, ABPA was detected in $25 \%$ to $37 \%$ of asthmatics with a positive skin prick test to Aspergillus fumigatus (Af) [11].

\section{Pathophysiology}

ABPA is caused by hypersensitivity to Aspergillus antigens [3]. In susceptible hosts, repeated inhalation of Aspergillus spores can cause an allergic response. This reaction is mainly an immunoglobulin E (IgE) mediated hypersensitivity reaction [3]. Both type III and type IV, immunoglobulin G (IgG) mediated immune complex and cell-mediated hypersensitivity reactions have also been seen [12]. ABPA is divided into five stages (i) acute, (ii) remission, (iii) exacerbation, (iv) corticosteroid-dependent asthma, and (v) fibrotic lung disease (Table 1) [1213]. The chest radiograph of the patient on admission (diagnosed with ABPA) and four months later is shown in Figures 1-2.

\section{Conventional Staging}

Stage I Acute: The patient is diagnosed with ABPA. All typical features such as aspergillus-specific IgE, radiological abnormalities (Figures 1-2), peripheral blood eosinophilia, and aspergillus-specific serum precipitins [3].

Stage II Remission: Asymptomatic patient with underlying controlled asthma with no new radiological infiltrates and no rise in total $\lg E$ for a minimum of six months [3].

Stage III Exacerbation: New pulmonary infiltrates appear on chest radiograph with peripheral blood eosinophilia and double the remission level IgE levels [3].

Stage IV Corticosteroid Dependent Asthma: Patients become dependent on corticosteroid treatment and are unable to completely taper off from them [3].

Stage V Fibrotic Lung Disease: Chest radiograph and CT scans will show irreversible fibrosis and chronic cavitation. Despite this, serological parameters are usually negative [3].

\section{TABLE 1: Conventional Staging in ABPA}

ABPA: allergic bronchopulmonary aspergillosis; IgE: immunoglobulin $\mathrm{E}$; $\mathrm{CT}$ : computed tomography. 


\section{Cureus}

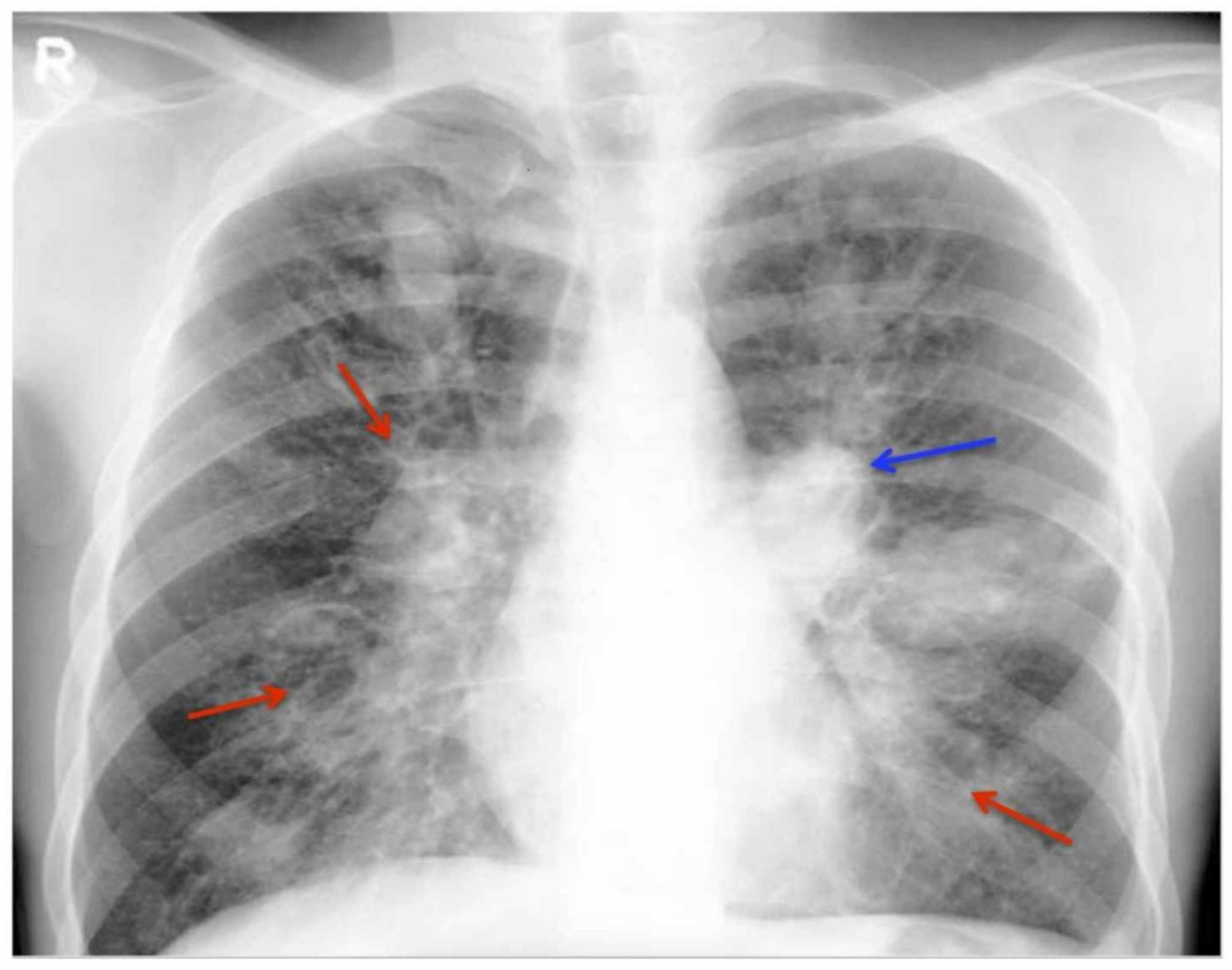

\section{FIGURE 1: Plain Chest Radiograph}

The chest radiograph of an allergic bronchopulmonary aspergillosis (ABPA) patient shown with leftsided perihilar opacity (blue arrow) along with non-homogeneous infiltrates (transient pulmonary infiltrates indicated by red arrows) in all zones of both lung fields, seen in acute and remission stage of ABPA [3]. 


\section{Cureus}

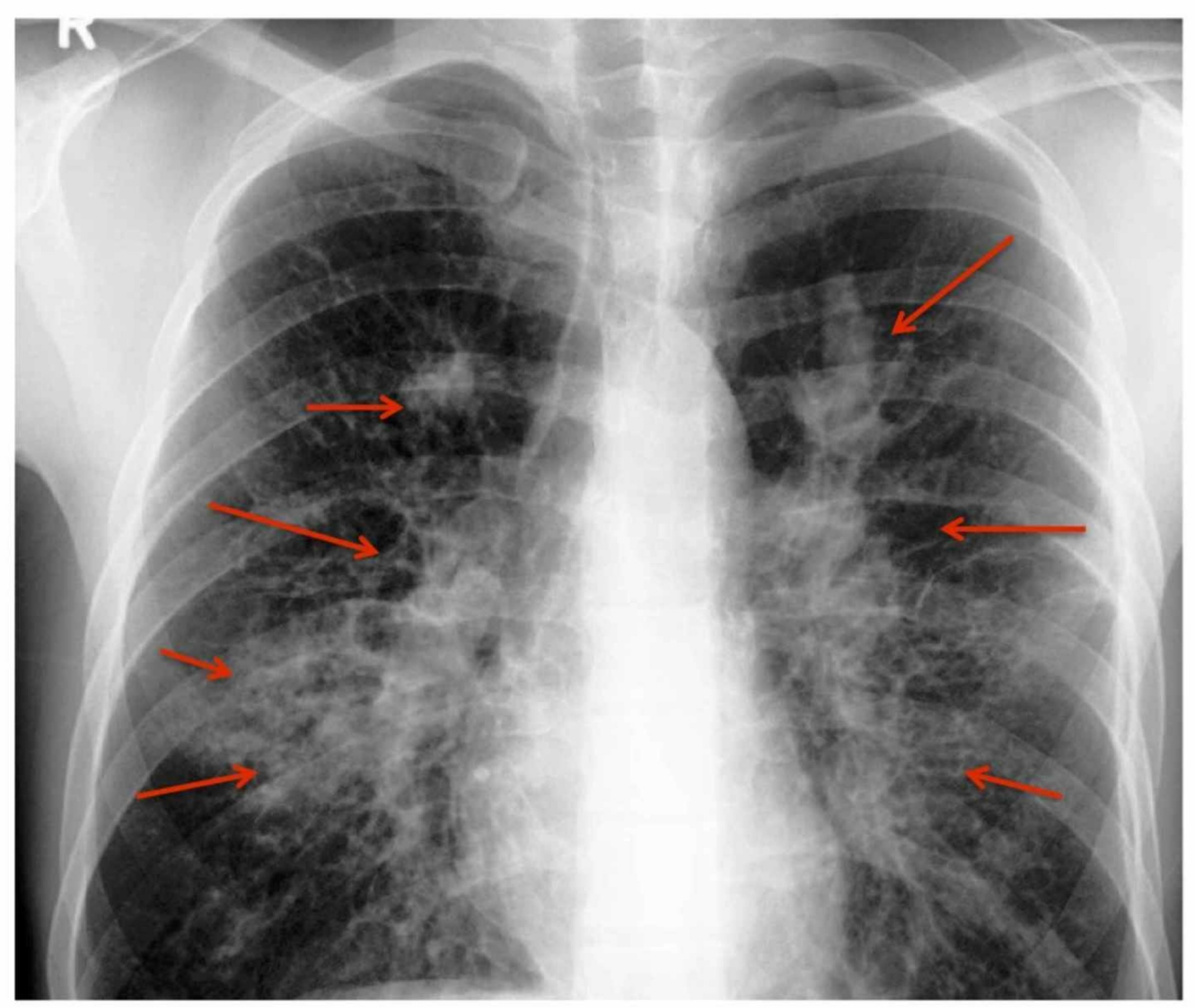

FIGURE 2: Chest Radiograph of the Same Patient Four Months

\section{Later}

Chest radiograph showed spontaneous resolution of left-sided perihilar opacity with an increase in non-homogenous infiltrates (red arrows) [3].

\section{Diagnostic criteria}

ABPA was first discovered by Hinson in 1952 when he reported and reviewed eight ABPA cases [14]. Years later in 1968, ABPA was first diagnosed in the United States and only afterwards became recognized on a global scale $[13,15]$. Over the years, the key diagnostic features have been standardized [15-16]. Because of the lack of consensus, there have been many proposed criteria over the years (Table 2). Although a set of criteria is required, there is no single test that establishes the diagnosis other than a demonstration of central bronchiectasis $(\mathrm{CB})$ with normal tapering bronchi, a feature still considered pathognomonic of ABPA (Figure 3) [15-17]. On high-resolution computed tomography (CT), high-attenuation mucous (HAM) plugs were also reported in $28 \%$ of patients with ABPA (Figure 4) [18]. The International Society for Human and Animal Mycology (ISHAM) working group has highlighted this finding and considers HAM a pathognomonic feature of ABPA [18]. 


\title{
Cureus
}

\author{
Name \\ Diagnostic Criteria for ABPA \\ 1977 , \\ Major Criteria: (1) asthma, (2) presence of fleeting or fixed pulmonary opacities on chest radiograph, (3) \\ Rosenberg- \\ immediate cutaneous hypersensitivity reaction to Af, (4) total serum IgE elevated, more than $1000 \mathrm{IU} / \mathrm{mL}$, \\ Patterson \\ Criteria $[15$, \\ 16] \\ (5) precipitating antibodies against Af, (6) peripheral blood eosinophilia, (7) central or proximal \\ bronchiectasis with normal tapering of distal bronchi \\ 1999, ABPA \\ in CF [19] \\ Minor Criteria: (1) golden brown sputum plugs in expectorant, (2) positive sputum culture for aspergillus \\ species, (3) late (arthus-type) skin reactivity to Af \\ Presence of two out of following three: (1) immediate cutaneous hypersensitivity reaction to Af, (2) \\ presence of precipitating antibodies to Af, (3) elevated total IgE levels more than $1000 \mathrm{IU} / \mathrm{mL}$ \\ Plus at least two of following six: (1) bronchoconstriction, (2) eosinophil count more than 1000/ $\mathrm{LL}$, (3) \\ history of pulmonary opacities on chest radiograph, (4) elevated IgE or IgG antibodies to Af, (5) Af in \\ sputum smear or sputum culture, (6) response to steroids
}

2002,

Minimum

Essential

Criteria [20]

Criteria: (1) asthma, (2) immediate cutaneous hypersensitivity reaction to Af, (3) total serum IgE elevated more than $1000 \mathrm{ng} / \mathrm{mL}$ (417 kU/L), (4) elevated IgE and IgG antibodies to Af, (5) CB in absence of distal bronchiectasis

2012,

Minimum Criteria: (1) patients with asthma or cystic fibrosis, (2) worsening lung function, (3) positive skin

Minimum

Criteria and

Additional prick test with aspergillus species, (4) total serum IgE greater than $1000 \mathrm{ng} / \mathrm{mL}(416 \mathrm{IU} / \mathrm{mL})$, (5) increased aspergillus species-specific IgE and IgG antibodies, (6) infiltrates noted on chest radiography

Criteria [4]

Additional Criteria: (1) increase in serum eosinophilia when the patient is not on corticosteroids ( more than 400 eosinophils $/ \mu \mathrm{L}),(2)$ aspergillus species-specific precipitating antibodies, (3) central bronchiectasis, (4) aspergillus species-specific containing mucus plugs

2013, Truly

Minimal

Criteria [21]

Criteria: (1) asthma, (2) immediate cutaneous hypersensitivity reaction to Af, (3) total serum IgE elevated more than $1000 \mathrm{ng} / \mathrm{mL}$ (417 kU/L), (4) CB in absence of distal bronchiectasis

Predisposing Conditions: (1) asthma, (2) CF

Obligatory Criteria (both need to be present): (1) type I aspergillus skin test positive (immediate

2013, ISHAM cutaneous hypersensitivity reaction to Af) or elevated IgE levels against Af, (2) elevated total IgE levels Working more than $1000 \mathrm{IU} / \mathrm{mL}$ (unless all other criteria is met, then total lgE levels can be less than $1000 \mathrm{IU} / \mathrm{mL}$ )

Group [22]

Other Criteria (two out of three at least): (1) presence of $\lg G$ antibodies against Af or precipitating antibodies, (2) presence of fleeting or fixed pulmonary opacities on chest radiograph consist with ABPA,

(3) eosinophil count more than 500 cells $/ \mu \mathrm{L}$ in steroid naïve patient (may be a historical value)

\section{TABLE 2: Diagnostic Criteria for ABPA}

ABPA: allergic bronchopulmonary aspergillosis; Af: Aspergillus fumigatus; CB: central bronchiectasis; CF: cystic fibrosis; IgE: immunoglobulin E; IgG: immunoglobulin G; ISHAM: International Society for Human and Animal Mycology. 


\section{Cureus}

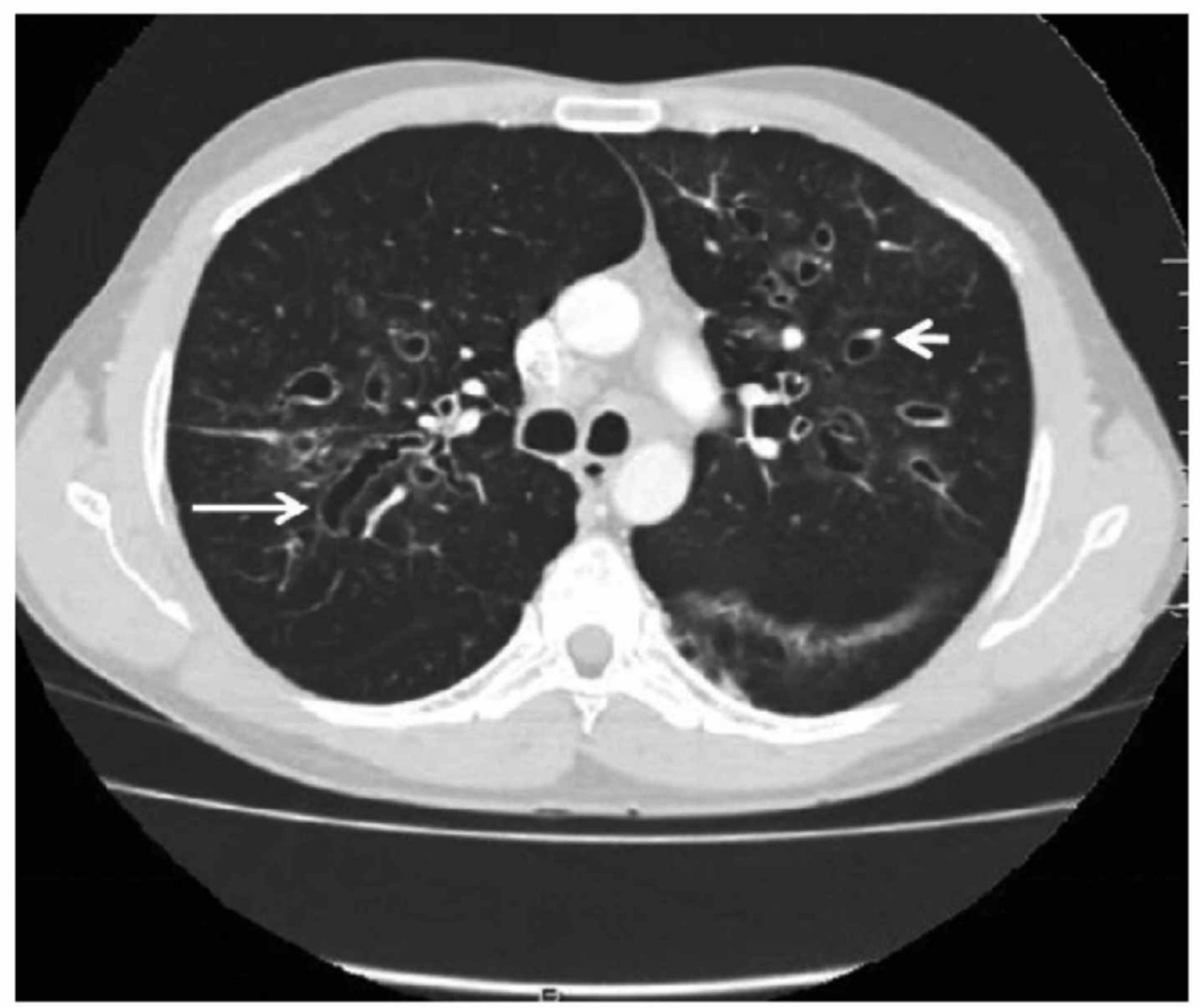

\section{FIGURE 3: Computed Tomography (CT) of the Thorax}

CT showing 'signet ring' (short, thick arrow) and 'string of pearls' (long, thin arrow) appearances, indicative of central bronchiectasis. Mucoid impaction and dilated bronchi are also seen [3]. 


\section{Cureus}
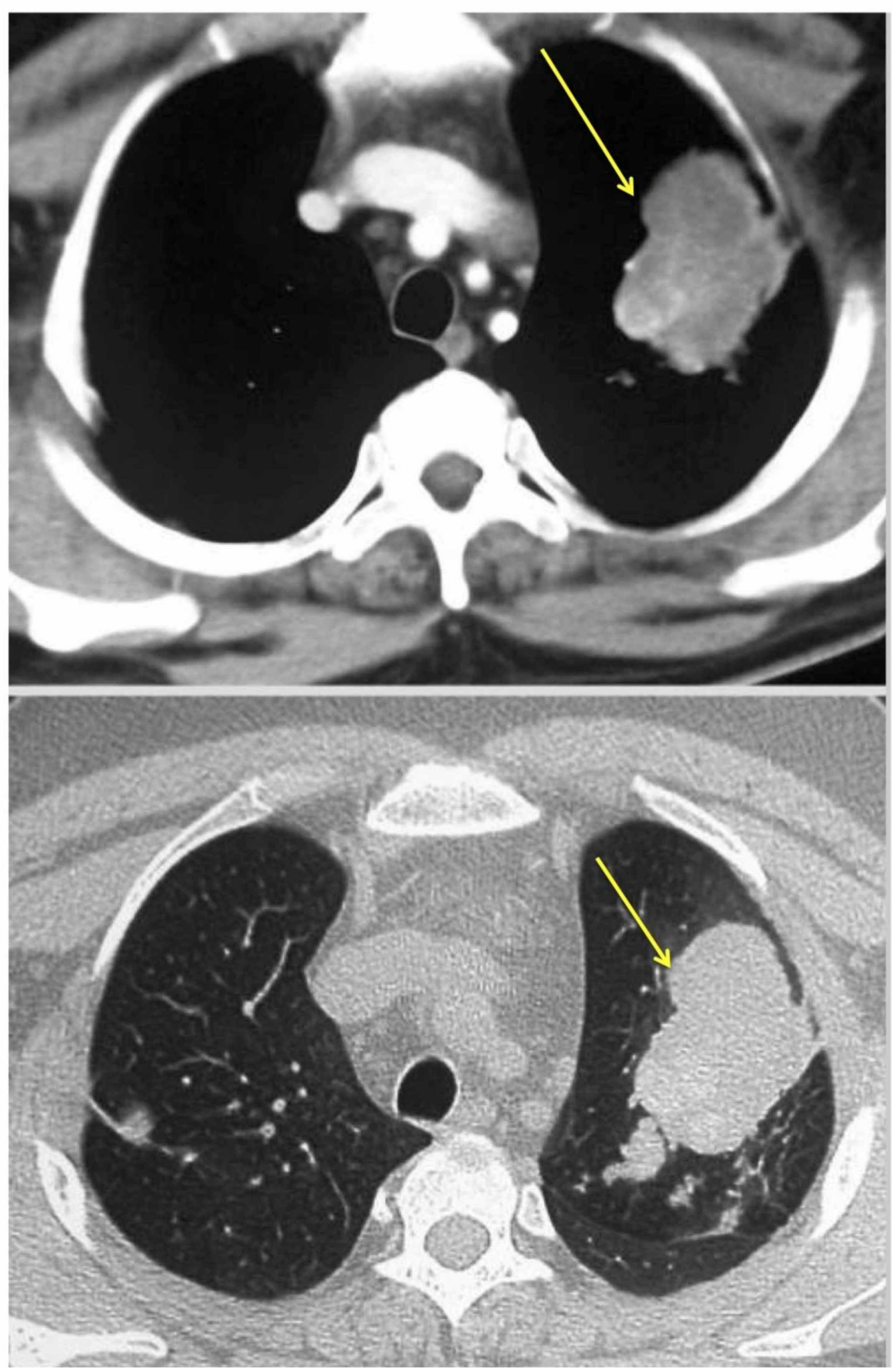

FIGURE 4: High-resolution Computed Tomography (HRCT) of the Thorax

HRCT of the thorax (mediastinal window and corresponding section on the lung window) showing high attenuation mucous (HAM) impaction (yellow arrow) [3]. This is considered pathognomic for allergic bronchopulmonary aspergillosis (ABPA) by the International Society for Human and Animal Mycology (ISHAM) working group [3]. 
In 1977, Rosenberg and Patterson proposed a diagnostic criteria divided into major and minor criteria. All eight major criteria may not be found at all times [3]. Some of the features may be present only during the acute (stage 1) or the exacerbation (stage 3) stages [3]. The major criteria consisted of patients diagnosed with asthma, the presence of pulmonary opacities on chest radiographs, immediate cutaneous reactivity to Af, the serum IgE being more than 1000 $\mathrm{IU} / \mathrm{mL}$, precipitating antibodies against Af, peripheral blood eosinophilia, and central or peripheral bronchiectasis with normal tapering of distal bronchi [15-16]. The minor criteria consisted of finding golden brown sputum plugs in expectorant, a positive sputum culture for Aspergillus species, and a late (arthus-type) skin reactivity to Af [15-16].

\section{ABPA in Cystic Fibrosis}

In 1999, Geller proposed a two-part diagnostic criteria. The first part needed two confirmed points out of three [19]. These were an immediate cutaneous reactivity to Af, the presence of precipitating antibodies to Af, and the elevated total IgE levels being more than $1000 \mathrm{IU} / \mathrm{mL}$ [19]. The second part needed two confirmed points out of the following six. These were bronchoconstriction, an eosinophil count more than $1000 / \mu \mathrm{L}$, a history of pulmonary opacities on chest radiograph, elevated IgE or IgG antibodies to Af, the presence of Af in sputum smears or cultures, and finally an observed response to steroids [19].

\section{Minimum Essential Criteria}

In 2002, Greenberger proposed a criteria that consisted of five points. These are a confirmed diagnosis of asthma, an immediate cutaneous reactivity to Af, a total serum IgE more than $1000 \mathrm{ng} / \mathrm{mL}(417 \mathrm{kU} / \mathrm{L})$, elevated IgE and IgG to Af, and central bronchiectasis in absence of distal bronchiectasis [20].

\section{Minimum Criteria and Additional Criteria}

In 2012, Knutsen updated the ABPA criteria with a minimum criteria and an additional criteria [4]. The minimum criteria consists of patients with asthma or cystic fibrosis, worsening lung function, a positive skin prick test with Aspergillus species, a total serum IgE greater than 1000 $\mathrm{ng} / \mathrm{mL}$ (416 IU/mL), increased Aspergillus species-specific IgE and IgG antibodies, and finally, infiltrates noted on the chest radiograph [4]. The additional criteria included an increase in serum eosinophilia when the patient is not on corticosteroids (more than 400 eosinophils/ $\mu \mathrm{L}$ ), Aspergillus species-specific precipitating antibodies, central bronchiectasis, and lastly, Aspergillus species-specific containing mucus plugs [4].

\section{Truly Minimal Criteria}

In 2013, Greenberger established a second set of diagnostic criteria. It contained four points [21]. They are a previous diagnosis of asthma, an immediate cutaneous reactivity to Af, an elevated serum IgE more than $1000 \mathrm{ng} / \mathrm{mL}$ (417 kU/L), and central bronchiectasis in absence of distal bronchiectasis [21].

\section{ISHAM Working Group}

In 2013, the ISHAM working group developed their own criteria for ABPA. It was divided into predisposing conditions like asthma or cystic fibrosis. The next section was the obligatory 
criteria which consisted of two points, both of which need to be present [22]. They are an immediate cutaneous reactivity to Af or elevated IgE levels directed against Af, and elevated total IgE levels more than $1000 \mathrm{IU} / \mathrm{mL}$ [22]. The other criteria consisted of three points, in which two of three must be present. They are the presence of IgG antibodies against Af, the presence of pulmonary opacities on chest radiograph, and lastly an eosinophil more than 500 cells/ $\mu \mathrm{L}$ in steroid naïve patient [22].

\section{Conclusions}

The material reviewed in this paper focuses on the diagnosis of ABPA. It goes into detail regarding the epidemiology, the pathophysiology, and the diagnostic criteria seen with ABPA. Despite these key points being addressed, more and larger studies are needed to create a standardized criteria that can be used worldwide to diagnose ABPA. Although there have been many proposed criteria over the years, it is imperative that a standardized one is created that can be utilized worldwide. This is a review article for busy, practicing physicians to have a cumulative view of our current situation regarding where the medical community is in our understanding of the diagnosis of ABPA.

\section{Additional Information}

\section{Disclosures}

Conflicts of interest: In compliance with the ICMJE uniform disclosure form, all authors declare the following: Payment/services info: All authors have declared that no financial support was received from any organization for the submitted work. Financial relationships: All authors have declared that they have no financial relationships at present or within the previous three years with any organizations that might have an interest in the submitted work. Other relationships: All authors have declared that there are no other relationships or activities that could appear to have influenced the submitted work.

\section{References}

1. Shah A: Allergic bronchopulmonary aspergillosis. Indian J Chest Dis Allied Sci. 1998, 40:4154.

2. deShazo RD, Chapin K, Swain RE: Fungal sinusitis. N Engl J Med. 1997, 337:254-259. 10.1056/NEJM199707243370407

3. Shah A, Panjabi C: Allergic bronchopulmonary aspergillosis: a perplexing clinical entity . Allergy Asthma Immunol Res. 2016, 8:282-97. 10.4168/aair.2016.8.4.282

4. Rodrigues J, Caruthers C, Azmeh R, Dykewicz MS, Slavin RG, Knutsen AP: The spectrum of allergic fungal diseases of the upper and lower airways. Expert Rev Clin Immunol. 2016, 12:531-50. 10.1586/1744666X.2016.1142874

5. Knutsen AP, Bush RK, Demain JG, et al.: Fungi and allergic lower respiratory tract diseases. J Allergy Clin Immunol. 2012, 2:280-91. 10.1016/j.jaci.2011.12.970

6. Douglass JA, Sandrini A, Holgate S: Allergic bronchopulmonary aspergillosis and hypersensitivity pneumonitis. Middleton's Allergy Principles and Practice. Adkinson NF Jr, Bruce Bochner A, Burks W (ed): Elsevier Saunders, Philadelphia; 2014. 1000-1007.

7. Agarwal R, Garg M, Aggarwal AN, Saikia B, Gupta D, Chakrabarti A: Serologic allergic bronchopulmonary aspergillosis (ABPA-S): long-term outcomes. Respir Med. 2012, 106:942-7. 10.1016/j.rmed.2012.03.001

8. Stevens DA, Moss RB, Kurup VP, et al.: Allergic bronchopulmonary aspergillosis in cystic fibrosis--state of the art: Cystic Fibrosis Foundation Consensus Conference. Clin Infect Dis. 2003, 37:225-264. 10.1086/376525

9. Denning DW, Pleuvry A, Cole DC: Global burden of allergic bronchopulmonary aspergillosis with asthma and its complication chronic pulmonary aspergillosis in adults. Med Mycol. 2013, 51:361-370. 10.3109/13693786.2012.738312

10. Greenberger PA, Patterson R: Allergic bronchopulmonary aspergillosis and the evaluation of 
the patient with asthma. J Allergy Clin Immunol. 1988, 81:646-50.

11. Shah A, Panjabi C: Allergic aspergillosis of the respiratory tract . Eur Respir Rev. 2014, 23:8-29. 10.1183/09059180.00007413

12. Greenberger PA, Patterson R: Diagnosis and management of allergic bronchopulmonary aspergillosis. Ann Allergy. 1986, 56:444-448.

13. Patterson R, Greenberger PA, Radin RC, Roberts M: Allergic bronchopulmonary aspergillosis: staging as an aid to management. Ann Intern Med. 1982, 96:286-291. 10.7326/0003-4819-963-286

14. Hinson KF, Moon AJ, Plummer NS: Broncho-pulmonary aspergillosis: a review and a report of eight new cases. Thorax. 1952, 7:317-33.

15. Rosenberg M, Patterson R, Mintzer R, Cooper BJ, Roberts M, Harris KE: Clinical and immunologic criteria for the diagnosis of allergic bronchopulmonary aspergillosis. Ann Intern Med. 1977, 86:405-414. 10.7326/0003-4819-86-4-405

16. Wang JL, Patterson R, Rosenberg M, Roberts M, Cooper BJ: Serum IgE and IgG antibody activity against Aspergillus fumigatus as a diagnostic aid in allergic bronchopulmonary aspergillosis. Am Rev Respir Dis. 1978, 117:917-927.

17. Panchal N, Pant C, Bhagat R, Shah A: Central bronchiectasis in allergic bronchopulmonary aspergillosis: comparative evaluation of computed tomography of the thorax with bronchography. Eur Respir J. 1994, 7:1290-1293. 10.1183/09031936.94.07071290

18. Goyal R, White CS, Templeton PA, Britt EJ, Rubin LJ: High attenuation mucous plugs in allergic bronchopulmonary aspergillosis: CT appearance. J Comput Assist Tomogr. 1992, 16:649-650.

19. Geller DE, Kaplowitz H, Light MJ, Colin AA: Allergic bronchopulmonary aspergillosis in cystic fibrosis: reported prevalence, regional distribution, and patient characteristics. Chest. 1999, 116:639-646. 10.1378/chest.116.3.639

20. Greenberger PA: Allergic bronchopulmonary aspergillosis. J Allergy Clin Immunol. 2002, 110:685-692. 10.1067/mai.2002.130179

21. Greenberger PA: When to suspect and work up allergic bronchopulmonary aspergillosis . Ann Allergy Asthma Immunol. 2013, 111:1-4. 10.1016/j.anai.2013.04.014

22. Agarwal R, Chakrabarti A, Shah A, et al.: Allergic bronchopulmonary aspergillosis: review of literature and proposal of new diagnostic and classification criteria. Clin Exp Allergy. 2013, 43:850-873. 10.1111/cea.12141 